\title{
Association of young age and male sex with primary sclerosing cholangitis in Taiwanese patients with inflammatory bowel disease
}

\author{
Meng-Tzu Weng ${ }^{1}$, I-Lun Shih ${ }^{2}$, Chien-Chih Tung ${ }^{3}$, Yew-Loong Leong ${ }^{1,4}$, Ming-Jium Shieh ${ }^{5}$, Cheng-Yi Wang ${ }^{1}$, \\ Jau-Min Wong ${ }^{1}$, Yen-Hsuan $\mathrm{Ni}^{6}$, Shu-Chen $\mathrm{Wei}^{1}$ \\ Departments of ${ }^{1}$ Internal Medicine, ${ }^{2}$ Medical Imaging, and ${ }^{3}$ Integrated Diagnostics and Therapeutics, National Taiwan University Hospital, \\ National Taiwan University College of Medicine, Taipei City; ${ }^{4}$ Good Liver Clinic, Taipei City; Departments of ${ }^{5}$ Oncology and ${ }^{6}$ Pediatrics, \\ National Taiwan University Hospital, National Taiwan University College of Medicine, Taipei City, Taiwan
}

Background/Aims: Primary sclerosing cholangitis (PSC) is associated with inflammatory bowel disease (IBD). We aimed to evaluate the prevalence, clinical manifestation, and outcomes of PSC in Taiwanese patients with IBD. Methods: This retrospective study enrolled patients with IBD admitted from January 1, 1996, to December 31, 2018, to National Taiwan University Hospital. A case-matched analysis was performed comparing patients with IBD with and without PSC according to age, sex, and time of admission, with ratios of 1:4 and 1:2 in the adult and pediatric groups, respectively. Results: In total, 763 patients with IBD were enrolled, 12 of whom were also diagnosed with PSC (1.57\%). All these patients had ulcerative colitis (UC). A greater incidence of IBD with PSC was observed in younger patients than in older patients. Male sex was a risk factor for PSC in pediatric patients with IBD $(P=0.015) ; 75 \%$ of these patients were diagnosed with PSC along with or after the diagnosis of UC. There was no significant difference in colitis extent and severity between the groups; however, a higher proportion of rectal sparing was observed in patients with PSC $(P=0.001)$. There was no significant difference in cancer development between the groups $(P=0.679)$. Conclusions: A $1.57 \%$ prevalence of PSC was observed in Taiwanese patients with IBD. The majority of patients with IBD and PSC were men and were diagnosed at a younger age. Hence, routine evaluation of biliary enzymes and liver imaging is recommended in young male patients with IBD. (Intest Res 2022;20:224-230)

Key Words: Primary sclerosing cholangitis; Inflammatory bowel disease; Ulcerative colitis

\section{INTRODUCTION}

Primary sclerosing cholangitis (PSC) is a chronic disease that affects the biliary tract. Progressive inflammation and fibrosis cause intrahepatic and extrahepatic bile duct obstruction and dilatation. The diagnosis of PSC is based on cholestatic biochemical profile and cholangiography. The annual incidence

Received March 12, 2021. Revised April 2, 2021. Accepted April 10, 2021. Correspondence to Shu-Chen Wei, Department of Internal Medicine, National Taiwan University Hospital, National Taiwan University College of Medicine, No. 7 Chung-Shan South Road, Taipei City 100, Taiwan. Tel: +8862-23123456 (ext. 65768), Fax: +886-2-23947927, E-mail: shuchenwei@ntu. edu.tw of PSC was 0.5-1.3 per 100,000 individuals in Western countries, ${ }^{1,2}$ whereas no relative information is available in Asian countries. The prevalence of PSC was approximately 0.0950.13 per 10,000 inhabitants in Asian countries, which is 10-fold lower than the prevalence in Western countries. ${ }^{3}$

Although the exact etiology and pathogenesis of PSC are still unknown, several studies have shown an association of PSC with certain genetic, environmental, and immunologic factors. There is no curative treatment for PSC, and a considerable number of patients develop severe clinical sequelae, such as portal hypertension, liver cirrhosis, and end-stage liver disease, eventually requiring liver transplantation (LT). Moreover, 
patients with PSC have a higher lifetime risk of cholangiocarcinoma and gall bladder cancer.

PSC is strongly associated with inflammatory bowel disease (IBD), especially ulcerative colitis (UC). Approximately 47\%$76 \%$ and $20 \%-37 \%$ of patients with PSC had IBD in Western and Asian countries, respectively. ${ }^{1}$ The proportion of UC was over $75 \%$ in these cases. ${ }^{4}$ By contrast, PSC was less commonly diagnosed in patients with IBD, occurring in approximately $2.4 \%-7.5 \%$ of patients with UC and approximately $3 \%$ of patients with Crohn's disease (CD) in Western countries. ${ }^{4}$ With regard to Asian countries, PSC was identified in $0.3 \%$ and $0.2 \%$ of Korean patients with UC and CD, respectively. ${ }^{5}$ Approximately $25 \%$ of patients with IBD and PSC developed PSC before the diagnosis of IBD. ${ }^{6}$

Patients with IBD and concomitant PSC were characterized by pancolitis, backwash ileitis, rectal sparing, and increased risk of colorectal cancer (CRC) and cholangiocarcinoma ${ }^{7-9}$ in Western countries, whereas the relationship between PSC and IBD was less frequently reported in Asian countries. Previously, both $\mathrm{IBD}^{10}$ and PSC $^{5,11}$ were less prevalent in Taiwan as well as in overall Asia. Therefore, limited data were available regarding PSC and IBD among Asians. With the increasing prevalence of IBD in Taiwan in recent decades, ${ }^{10}$ we sought to evaluate possible factors associated with PSC that can aid earlier diagnosis of PSC in IBD. Compared with patients with IBD and without PSC, those with concomitant IBD and PSC have a higher risk of hepatic failure. Earlier identification and preparation for LT might be helpful for improving outcomes in such patients.

\section{METHODS}

\section{Patients}

This retrospective study was conducted to review patients with IBD admitted to National Taiwan University Hospital from January 1, 1996, to December 31, 2018. This study was approved by the Institutional Review Board of National Taiwan University Hospital (IRB No. 201912236RINC). This study is a retrospective study using anonymous clinical data review and so informed consent was waived. The International Classification of Diseases, Ninth Revision, Clinical Modification (ICD-9-CM) codes were used for the detection of UC (ICD-9-CM code 556.9) and CD (ICD-9-CM code 555.9). The type of IBD and the extent and severity of colitis were recorded. The diagnosis of IBD was based on a combination of clinical, endoscopic, and histologic features and the exclusion of an infectious etiol- ogy. The extent of colitis was classified as proctitis (E1), left sided (E2), and extensive (E3) in $\mathrm{UC}^{12}$ according to the Montreal classification. The severity of disease (mild, moderate, or severe) was determined according to Truelove and Witts criteria. ${ }^{8}$ Patients with IBD with concomitant PSC were identified from the radiology information system of National Taiwan University Hospital. All types of radiological reports with the keyword "sclerosing" and "cholangitis" were searched, and the charts of patients who repeatedly underwent computed tomography, magnetic resonance imaging, or percutaneous biliary procedures were reviewed. The diagnostic criteria of PSC were based on cholestatic biochemical profile and cholangiography. Studies involving patients with IgG4-associated cholangitis and secondary causes of sclerosing cholangitis were excluded. Clinical information, including demographic data, laboratory and pathologic reports, treatment regimens, the area involved in PSC, the disease course, and a history of cancer or LT, was assessed. A total of 12 patients with concomitant IBD and PSC were identified. Adult patients with IBD and PSC were matched with those with IBD but without PSC, as a comparison group, with a 1:4 ratio, according to sex, age, and time of hospital admission. Because the number of patients in the pediatric group (patients aged below 18 years) was small, pediatric patients with IBD and PSC were matched with those with IBD but without PSC in a 1:2 ratio. Differences in clinical information and outcomes between patients with IBD and PSC and those with IBD but without PSC were compared.

\section{Statistical Analysis}

Continuous variables are expressed as the mean \pm standard deviation. Categorical variables are expressed as the frequency (percentage). Student $t$-test was used to compare quantitative variables, and the chi-square statistic or Fisher exact test was used to compare categorical variables between the $2 \mathrm{Co}^{-}$ horts. A $P$-value less than 0.05 was considered statistically significant. Statistical analyses were performed using SPSS 11.0 program (SPSS Inc., Chicago, IL, USA).

\section{RESULTS}

\section{Demographic Characteristics of Patients with UC}

A total of 763 patients were diagnosed with IBD (380 with CD and 383 with UC); 12 of them with UC had concomitant PSC. The incidence of PSC was $1.57 \%$ in patients with IBD and $3.1 \%$ in patients with UC. Patients with UC and PSC were significantly younger than those with UC but without PSC (median 
age: 22.5 and 49.0 years, respectively; $P=0.001)$. Male predominance was noted in both the groups $(83.3 \%$ and $58.5 \%$, respectively; $P=0.085$ ). After the subgroup analysis of pediatric patients with UC, a significantly higher proportion of male patients was observed in the PSC group than in the non-PSC group ( $100 \%$ and $38.2 \%$, respectively; $P=0.015)$. The demographics of patients with IBD with and without PSC are summarized in Table 1. The basic characteristics of 12 patients with UC and PSC are listed in Table 2. The majority (75\%) of these patients were diagnosed with PSC along with or after the diagnosis of UC. The typical PSC magnetic resonance imaging images were shown in Supplementary Fig. 1.

Table 1. Basic Characteristics of Patients with UC and PSC and Those with UC but without PSC

\begin{tabular}{lccc}
\hline Characteristics & PSC UC & Non-PSC UC & $P$-value \\
\hline Age $(y r)$ & $22.5(12-27)$ & $49.0(32-59)$ & $0.001^{\text {a }}$ \\
Male sex & $10 / 12(83.3)$ & $217 / 371(58.5)$ & 0.085 \\
Age $\leq 16 \mathrm{yr}$ & $5 / 5(100)$ & $13 / 34(38.2)$ & $0.015^{\mathrm{a}}$ \\
Age $>16 \mathrm{yr}$ & $5 / 7(71.4)$ & $204 / 337(60.5)$ & 0.560 \\
\hline
\end{tabular}

Values are presented as median (interquartile) or number/number (\%). aStatistically significant, $P<0.05$. Fisher exact test was used for small samples, and Student $t$-test was used for quantitative variables. Chi-square test was used for categorical variables.

PSC, primary sclerosing cholangitis; UC, ulcerative colitis.

Table 2. Basic Characteristics of 12 Patients with IBD (UC) and PSC

\begin{tabular}{|c|c|c|c|c|}
\hline $\begin{array}{l}\text { Patient } \\
\text { No. }\end{array}$ & $\begin{array}{l}\text { Age } \\
\text { (yr) }\end{array}$ & Sex & PSC type & $\begin{array}{c}\text { Time sequence of IBD } \\
\text { and PSC diagnosis }\end{array}$ \\
\hline 1 & 3 & $M$ & IHBD+EHBD & At the same time \\
\hline 2 & 5 & M & IHBD+EHBD & IBD after PSC \\
\hline 3 & 5 & M & IHBD+EHBD & At the same time \\
\hline 4 & 12 & $M$ & IHBD & IBD before PSC \\
\hline 5 & 15 & M & IHBD & At the same time \\
\hline 6 & 21 & $F$ & IHBD & IBD after PSC \\
\hline 7 & 24 & M & IHBD+EHBD & IBD before PSC \\
\hline 8 & 25 & M & IHBD+EHBD & IBD after PSC \\
\hline 9 & 27 & $M$ & IHBD+EHBD & IBD before PSC \\
\hline 10 & 42 & $\mathrm{~F}$ & IHBD+EHBD & At the same time \\
\hline 11 & 62 & M & IHBD+EHBD & IBD before PSC \\
\hline 12 & 71 & $M$ & $\mathrm{HBBD}+\mathrm{EHBD}$ & IBD before PSC \\
\hline
\end{tabular}

IBD, inflammatory bowel disease; UC, ulcerative colitis; PSC, primary sclerosing cholangitis; $M$, male; $F$, female; IHBD, intrahepatic bile duct; $E H B D$, extrahepatic bile duct.

\section{Comparison of Clinical Characteristics between Patients with UC with and without PSC}

Among the 12 patients who had concomitant UC and PSC, 7 were adults. They were matched with patients with UC but

Table 3. Comparison of Clinical Characteristics between Patients with UC and PSC and Matched Patients with UC but without PSC

\begin{tabular}{|c|c|c|c|}
\hline Variable & $\begin{array}{l}\text { PSC UC } \\
(n=12)\end{array}$ & $\begin{array}{c}\text { Non-PSC } \\
\text { UC }(n=38)\end{array}$ & $P$-value \\
\hline \multicolumn{4}{|l|}{ Underlying diseases } \\
\hline Hypertension & $1(8.3)$ & $2(5.3)$ & 0.696 \\
\hline Diabetes mellitus & 0 & $2(5.3)$ & 0.417 \\
\hline Hyperlipidemia & 0 & $1(2.6)$ & 0.570 \\
\hline Coronary artery disease & 0 & $2(5.3)$ & 0.417 \\
\hline Chronic kidney disease & $1(8.3)$ & $2(5.3)$ & 0.696 \\
\hline HBV carrier & 0 & 0 & - \\
\hline HCV carrier & 0 & $2(5.3)$ & 0.404 \\
\hline Colitis location/extent & & & 0.271 \\
\hline Proctitis & $2(16.7)$ & $4(10.5)$ & \\
\hline Left side colon & $1(8.3)$ & $12(31.6)$ & \\
\hline Extensive & $9(75.0)$ & $22(57.9)$ & \\
\hline Rectal sparing & $3(25.0)$ & 0 & $0.001^{\mathrm{a}}$ \\
\hline Backwash ileitis & 2/6 (33.3) & 2/22 (9.1) & 0.133 \\
\hline Disease severity & & & 0.513 \\
\hline Mild & $6(50.0)$ & 15 (39.5) & \\
\hline Moderate & $2(16.7)$ & $13(34.2)$ & \\
\hline Severe & $4(33.3)$ & $10(26.3)$ & \\
\hline \multicolumn{4}{|l|}{ Liver involvement } \\
\hline Jaundice & $6(50.0)$ & $1(2.6)$ & $<0.001^{a}$ \\
\hline Liver cirrhosis & $5(41.7)$ & $1(2.6)$ & $0.001^{\mathrm{a}}$ \\
\hline Hepatic decompensation & $3(25.0)$ & $1(2.6)$ & $0.013^{\mathrm{a}}$ \\
\hline Transplantation & $3(25.0)$ & 0 & $0.001^{\mathrm{a}}$ \\
\hline \multicolumn{4}{|l|}{ Treatment } \\
\hline UDCA & $12(100)$ & 0 & $0.001^{\mathrm{a}}$ \\
\hline 5-ASA & 10 (83.3) & $38(100)$ & 0.054 \\
\hline Steroid & $7(58.3)$ & $20(52.6)$ & 0.730 \\
\hline Thiopurine & $5(41.7)$ & $18(47.4)$ & 0.730 \\
\hline Anti-TNF- $\alpha$ & 0 & $8(21.1)$ & 0.083 \\
\hline Cancer & $1(8.3)$ & $3(7.9)$ & 0.679 \\
\hline Mortality & $2(16.7)$ & $3(7.9)$ & 0.377 \\
\hline
\end{tabular}

Values are presented as number (\%) or number/number (\%).

${ }^{a}$ Statistically significant, $P<0.05$. Chi-square test was used for categorical variables.

UC, ulcerative colitis; PSC, primary sclerosing cholangitis; HBV, hepatitis $B$ virus; $\mathrm{HCV}$, hepatitis $C$ virus; UDCA, ursodeoxycholic acid; 5-ASA, 5-aminosalicylic acid, TNF, tumor necrosis factor. 
without PSC according to sex, age, and the year of admission as a control group, at a 1:4 ratio in the adult group and 1:2 ratio in the pediatric group. Finally, a total of 38 matched patients (28 adults and 10 children) with UC were included as controls. The clinical characteristics of these patients are listed in Table 3. Underlying diseases, namely hypertension ( $8.3 \%$ vs. $5.3 \%$, $P=0.696$ ), diabetes mellitus ( $0 \%$ vs. $5.3 \%, P=0.417$ ), hyperlipidemia ( $0 \%$ vs. $2.6 \%, P=0.570)$, coronary artery disease $(0 \%$ vs. $5.3 \%, P=0.417$ ), chronic kidney disease ( $8.3 \%$ vs. $5.3 \%, P=0.696$ ), and chronic viral hepatitis, were comparable between the groups (all $P>0.05$ ). Moreover, there was no statistical difference in the colitis-involved area and disease severity between the groups, whereas patients with UC and PSC had higher proportion of rectal sparing, compared with patients with UC but without PSC (25\% and $0 \%$, respectively; $P=0.001$ ). The frequency of backwash ileitis did not differ between the 2 groups (33.3\% vs. $9.1 \%, P=0.133)$. However, the endoscope reached the terminal ileum in only 28 of 50 patients (6 in the PSC group and 22 in the non-PSC group). Furthermore, compared with patients in the non-PSC group, those in the PSC group presented more severe liver-associated symptoms and imaging findings. Patients in the PSC group had higher risks of jaundice $50.0 \%$ and $2.6 \%$, respectively; $P<0.001)$, liver cirrhosis $(41.7 \%$ and $2.6 \%$, respectively; $P<0.001)$, hepatic decompensation (25.0\% and $2.6 \%$, respectively; $P=0.013)$, and requiring LT ( $25.0 \%$ and $0 \%$, respectively; $P=0.001$ ) than did patients in the non-PSC group. Among the 3 patients who received LT for the PSC, 1 patient passed away 7 years after LT due to septic shock. All of them, their UC disease activity were stable during the followup period. With regarding to treatment, all patients with UC and PSC received ursodeoxycholic acid ( $100 \%$ vs. $0 \%, P<0.001$ ), whereas those without PSC did not. Otherwise, the frequency of administering 5 -aminosalicylic acid ( $83.3 \%$ vs. $100 \%, P=0.054$ ), steroids ( $58.3 \%$ vs. $52.6 \%, P=0.730$ ), thiopurine ( $41.7 \%$ vs. $47.4 \%$, $P=0.730)$, and anti-tumor necrosis factor- $\alpha(0 \%$ vs. $21.0 \%, P=$ 0.083 ) did not significantly differ between the groups. Patients with UC and PSC did not have a significantly higher risk of cancer ( $8.3 \%$ vs. $7.9 \%, P=0.679$ ) or higher mortality ( $16.7 \%$ vs. $7.9 \%, P=0.377$ ) than did patients with UC but without PSC. In the PSC-UC group, 1 patient developed colon cancer and 2 patients died (1 patient owing to hepatic failure and the other owing to colon cancer). In the non-PSC-UC group, 2 patients developed colon cancer and 1 patient developed acute myeloid leukemia. Three patients died (1 owing to HCV-related hepatic failure, 1 owing to colon cancer, and 1 owing to pneumonia), all of whom were aged over 60 years. The mortality
Table 4. Comparison of Laboratory Data between Patients with UC and PSC and Matched Patients with UC but without PSC

\begin{tabular}{lccc}
\hline Parameter & $\begin{array}{c}\text { PSC UC } \\
(\mathrm{n}=12)\end{array}$ & $\begin{array}{c}\text { Non-PSC UC } \\
(\mathrm{n}=38)\end{array}$ & P-value \\
\hline WBC $\left(\times 10^{3} / \mu \mathrm{L}\right)$ & $6,145 \pm 2,698$ & $6,800 \pm 3,493$ & 0.956 \\
$\mathrm{Hb}(\mathrm{g} / \mathrm{dL})$ & $10.60 \pm 1.92$ & $12.40 \pm 2.15$ & $0.002^{\mathrm{a}}$ \\
T-Bil $(\mathrm{mg} / \mathrm{dL})$ & $1.26 \pm 3.40$ & $0.54 \pm 0.52$ & $0.001^{\mathrm{a}}$ \\
ALT $(\mathrm{U} / \mathrm{L})$ & $73.00 \pm 172.40$ & $12.00 \pm 16.59$ & $<0.001^{\mathrm{a}}$ \\
ALP $(\mathrm{U} / \mathrm{L})$ & $275.50 \pm 231.50$ & $75.50 \pm 99.00$ & $<0.001^{\mathrm{a}}$ \\
$\gamma$-GT $(\mathrm{U} / \mathrm{L})$ & $165.00 \pm 111.70$ & $24.50 \pm 108.10$ & $0.005^{\mathrm{a}}$ \\
PT $(\mathrm{sec})$ & $11.40 \pm 5.36$ & $10.90 \pm 1.01$ & $0.042^{\mathrm{a}}$ \\
Alb $(\mathrm{g} / \mathrm{dL})$ & $3.65 \pm 0.53$ & $4.25 \pm 1.31$ & 0.092 \\
Cre $(\mathrm{mg} / \mathrm{dL})$ & $0.60 \pm 2.10$ & $0.75 \pm 0.44$ & 0.246 \\
CRP $(\mathrm{mg} / \mathrm{dL})$ & $1.51 \pm 1.92$ & $0.29 \pm 2.70$ & 0.626 \\
\hline
\end{tabular}

Value are presented as mean \pm standard deviation.

aStatistically significant, $P<0.05$. Student $t$-test was used for quantitative variables.

UC, ulcerative colitis; PSC, primary sclerosing cholangitis; WBC, white blood cells; $\mathrm{Hb}$, hemoglobin; T-Bil, total bilirubin; ALT, alanine aminotransferase; ALP, alkaline phosphatase; $\gamma$-GT, gamma-glutamyl transpeptidase; $\mathrm{PT}$, prothrombin time; Alb, albumin; Cre, creatinine; CRP, C-reactive protein.

rate did not significantly differ between the pediatric and adult groups ( $8.6 \%$ vs. $13.3 \%, P=0.629)$.

\section{Comparison of Laboratory Characteristics between Patients with and without PSC}

Compared with patients with UC but without PSC, those with UC and PSC had higher levels of serum total bilirubin (0.54 and $1.26 \mathrm{mg} / \mathrm{dL}$, respectively; $P=0.001$ ), alanine aminotransferase (12 and $73 \mathrm{U} / \mathrm{L}$, respectively; $P<0.001$ ), alkaline phosphatase (75.5 and $275.5 \mathrm{U} / \mathrm{L}$, respectively; $P<0.001$ ), $\gamma$-glutamyl transpeptidase (24.5 and $165 \mathrm{U} / \mathrm{L}$, respectively; $P=0.005$ ), and prothrombin time (10.9 and 11.4 seconds, respectively; $P=0.042$ ). Furthermore, the hemoglobin level was significantly lower in the PSC-UC group than in the non-PSC-UC group (10.6 and $12.4 \mathrm{~g} / \mathrm{dL}$, respectively; $P=0.002$ ). The relevant data are listed in Table 4

\section{DISCUSSION}

In this hospital-based cohort study, $1.57 \%$ prevalence of PSC was observed among Taiwanese patients with IBD; the prevalence was $3.1 \%$ in patients with UC and $0 \%$ in those with CD. Thus, all these IBD patients were diagnosed with UC. This finding is compatible with that of our previously published nationwide population-based study that showed a $1.6 \%$ prevalence 
of cholangitis in Taiwanese patients with IBD. ${ }^{13}$ The prevalence of PSC in Indian patients with UC and CD was $0.4 \%$ and $0 \%$, respectively, ${ }^{14}$ and that in Korean patients was $0.3 \%$ and $0.2 \%$, respectively. ${ }^{5}$ Meanwhile, $2.4 \%$ to $7.5 \%$ of patients with UC were diagnosed with PSC in Western countries. ${ }^{4}$ The lower prevalence of PSC along with IBD in Asian countries than in Western countries may be attributed to underdiagnosis; alternatively, the prevalence could have actually been low. To the best of our knowledge, PSC was more common among patients with IBD in Taiwan than in other Asian countries.

We observed that patients with IBD and PSC were much younger than those with IBD but without PSC; in addition, men were more likely to develop PSC, especially in the pediatric group. Male predominance of PSC in children and adolescents was previously reported. ${ }^{15}$ According to Vavricka et al., $25 \%$ of patients with IBD and PSC developed PSC before the diagnosis of IBD, which indicates that in the majority of cases, PSC would be diagnosed after the diagnosis of IBD. Our results also demonstrated that in $25 \%$ of patients, PSC was diagnosed before IBD, in $33 \%$ of patients, PSC and IBD were simultaneously diagnosed, and in $42 \%$ of patients, PSC was diagnosed after IBD. Interestingly, in the majority of pediatric patients (60\%), PSC and UC were simultaneously diagnosed. Our study also revealed that patients with UC and PSC had a higher risk of liver decompensation and increased requirement for LT. Therefore, routine survey and regular follow-up of liver and biliary profile function are recommended in pediatric male patients with IBD for early diagnosis and accurate management of PSC.

Our data demonstrated that underlying systemic disease or the extent or severity of colitis did not significantly differ between the PSC-UC and non-PSC-UC groups. Compared with patients with IBD alone, those with IBD and PSC have been reported to show predominant extensive colitis. ${ }^{16,17}$ Even in the current study, patients with IBD and PSC presented with more extensive colitis (75\%). Approximately $57.9 \%$ of patients in our matched control group presented with extensive colitis, whereas another hospital-based study conducted in Taiwan demonstrated that left-sided colitis was the predominant disease (42.7\%), followed by extensive colitis (39.1\%), in patients with UC. ${ }^{18}$ There was no difference in the area of colitis between the 2 patient groups in this study, which may be attributed to the fact that our control group patients had more extensive colitis, as our hospital was a referral center for this condition. More rectal-sparing type (RST) UC was identified in our PSC group than in the non-PSC group ( $25 \%$ and $0 \%$, respectively;
$P=0.001)$. In the study conducted by Park et al., ${ }^{19}$ RST was detected in 3.3\% of Korean patients with UC. RST was a relative disease status and could develop during disease progression. ${ }^{20}$ Currently, it is well established that RST UC is associated with PSC. ${ }^{21}$ Backwash ileitis was also reported as a distinct phenotype of patients with IBD and PSC..$^{22}$ In our study, although the proportion of ileitis seems higher in the group of patients with PSC, there was no statistical difference between the groups as terminal ileum intubation was achieved only in 28 patients (56\%). Complete ileocolonoscopy was recommended for all patients with an initial diagnosis of IBD.

Compared with patients with UC but without PSC, those with PSC had higher levels of bilirubin, liver enzymes, biliary enzymes, and prothrombin time and a lower level of hemoglobin. The majority of these laboratory profiles were associated with liver reserve and could be prognostic factors for hepatic failure. Thus, it is reasonable that these patients with PSC had higher risks of liver cirrhosis and hepatic failure and were more likely to require LT.

In the PSC-UC group, 1 patient developed colon cancer, whereas in the non-PSC-UC group, 2 patients developed colon cancer. UC was associated with a 2- to 4-fold increased risk of CRC. ${ }^{23}$ Compared with patients with IBD alone, those with IBD and PSC had an increased risk of CRC (odds ratio, 3.41; 95\% confidence interval, 2.13-5.48). ${ }^{24}$ The low rate of cancer in the PSC-UC group might be attributed to the small number of patients in this group. We recommend annual surveillance colonoscopy for patients with IBD and PSC according to guidelines. ${ }^{25}$

This study has some limitations. First, this was a retrospective study. Although we speculated that data regarding comprehensive laboratory studies would be available for hospitalized patients, not all patients had complete laboratory profiles or underwent cholangiography; hence, the prevalence of PSC might have been underestimated. Second, owing to the limited number of pediatric patients with IBD, the ratio of the PSC group to the control group was 1:2. The small patient number also led to a nonsignificant statistical result. Despite these limitations, the findings of this study help understand the characteristics of PSC concomitant with IBD in patients from Asian countries.

In conclusion, $1.57 \%$ prevalence of PSC was observed in Taiwanese patients with IBD, which was the highest in Asia. Pediatric male patients with UC had a higher risk of PSC. In the majority of patients, PSC was diagnosed along with or after IBD. Compared with patients with UC but without PSC, 
those with UC and PSC had higher levels of liver and biliary enzymes and higher risks of liver cirrhosis and hepatic failure. Routine survey and regular follow-up of hepatic and biliary profile function are recommended in pediatric male patients with IBD for early diagnosis and proper management of PSC to improve the outcomes of these patients.

\section{ADDITIONAL INFORMATION}

\section{Funding Source}

This work was supported by the National Taiwan University Hospital (NTUH 107-A139 to Wei SC) and the Liver Disease Prevention and Treatment Research Foundation, Taiwan (to Wei SC and Weng MT).

\section{Conflict of Interest}

Wong JM and Wei SC are editorial board members of the journal but were not involved in the peer reviewer selection, evaluation, or decision process of this article. No other potential conflicts of interest relevant to this article were reported.

\section{Author Contribution}

Drafting of the manuscript: Weng MT, Wei SC. Analysis and interpretation of the data: Weng MT, Shih IL, Wong JM. Acquisition of the data: Shih IL, Tung CC, Leong YL. Statistical analysis: Weng MT. Critical revision of the manuscript for important intellectual content and study supervision: Shieh MJ, Wang CY, Wong JM. Study concept and design: Wei SC, Ni YH. Approval of final manuscript: all authors.

\section{ORCID}

Weng MT

https://orcid.org/0000-0002-4143-6607

Shih IL

Tung CC

https://orcid.org/0000-0001-9187-1562

Leong YL

https://orcid.org/0000-0002-5468-9064

Shieh MJ

https://orcid.org/0000-0001-7512-1163

Wang CY

https://orcid.org/0000-0003-2921-4443

Wong JM

https://orcid.org/0000-0002-3698-8319

$\mathrm{Ni} \mathrm{YH}$

https://orcid.org/0000-0002-8971-3001

Wei SC

https://orcid.org/0000-0002-1158-5249

https://orcid.org/0000-0002-5017-5840

\section{Supplementary Material}

Supplementary materials are available at the Intestinal Research website (https://www.irjournal.org).

\section{REFERENCES}

1. Boonstra K, Beuers U, Ponsioen CY. Epidemiology of primary sclerosing cholangitis and primary biliary cirrhosis: a systematic review. J Hepatol 2012;56:1181-1188.

2. Boonstra K, Weersma RK, van Erpecum KJ, et al. Populationbased epidemiology, malignancy risk, and outcome of primary sclerosing cholangitis. Hepatology 2013;58:2045-2055.

3. Karlsen TH, Folseraas T, Thorburn D, Vesterhus M. Primary sclerosing cholangitis: a comprehensive review. J Hepatol 2017; 67:1298-1323.

4. de Vries AB, Janse M, Blokzijl H, Weersma RK. Distinctive inflammatory bowel disease phenotype in primary sclerosing cholangitis. World J Gastroenterol 2015;21:1956-1971.

5. Yang BR, Choi NK, Kim MS, et al. Prevalence of extraintestinal manifestations in Korean inflammatory bowel disease patients. PLoS One 2018;13:e0200363.

6. Vavricka SR, Rogler G, Gantenbein C, et al. Chronological order of appearance of extraintestinal manifestations relative to the time of IBD diagnosis in the Swiss Inflammatory Bowel Disease Cohort. Inflamm Bowel Dis 2015;21:1794-1800.

7. Folseraas T, Boberg KM. Cancer risk and surveillance in primary sclerosing cholangitis. Clin Liver Dis 2016;20:79-98.

8. Truelove SC, Witts LJ. Cortisone in ulcerative colitis; preliminary report on a therapeutic trial. Br Med J 1954;2:375-378.

9. Núñez F P, Quera P R, Gomollón F. Primary sclerosing cholangitis and inflammatory bowel disease: intestine-liver interrelation. Gastroenterol Hepatol 2019;42:316-325.

10. Yen HH, Weng MT, Tung CC, et al. Epidemiological trend in inflammatory bowel disease in Taiwan from 2001 to 2015: a nationwide populationbased study. Intest Res 2019;17:54-62.

11. Wei SC, Shieh MJ, Chang MC, Chang YT, Wang CY, Wong JM. Long-term follow-up of ulcerative colitis in Taiwan. J Chin Med Assoc 2012;75:151-155.

12. Dignass A, Eliakim R, Magro F, et al. Second European evidencebased consensus on the diagnosis and management of ulcerative colitis part 1: definitions and diagnosis. J Crohns Colitis 2012;6:965-990.

13. Lin WC, Weng MT, Tung CC, et al. Trends and risk factors of mortality analysis in patients with inflammatory bowel disease: a Taiwanese nationwide population-based study. J Transl Med 2019;17:414.

14. Singh B, Kedia S, Konijeti G, et al. Extraintestinal manifestations of inflammatory bowel disease and intestinal tuberculosis: frequency and relation with disease phenotype. Indian J Gastroenterol 2015;34:43-50. 
15. Fagundes ED, Ferreira AR, Hosken CC, Queiroz TC. Primary sclerosing cholangitis in children and adolescents. Arq Gastroenterol 2017;54:286-291.

16. Fousekis FS, Theopistos VI, Mitselos IV, et al. Specific features of patients with inflammatory bowel disease and primary sclerosing cholangitis. J Clin Med Res 2019;11:81-88.

17. Bajer L, Wohl P, Drastich P. PSC-IBD: specific phenotype of inflammatory bowel disease associated with primary sclerosing cholangitis. Vnitr Lek 2018;64:659-664.

18. Chou JW, Lai HC, Chang CH, Cheng KS, Feng CL, Chen TW. Epidemiology and clinical outcomes of inflammatory bowel disease: a hospital-based study in central Taiwan. Gastroenterol Res Pract 2019;2019:4175923.

19. Park SH, Yang SK, Park SK, et al. Atypical distribution of inflammation in newly diagnosed ulcerative colitis is not rare. Can J Gastroenterol Hepatol 2014;28:125-130.

20. Horio Y, Uchino M, Bando T, et al. Rectal-sparing type of ulcerative colitis predicts lack of response to pharmacotherapies. BMC Surg 2017;17:59.
21. Ricciuto A, Kamath BM, Griffiths AM. The IBD and PSC phenotypes of PSC-IBD. Curr Gastroenterol Rep 2018;20:16.

22. Palmela C, Peerani F, Castaneda D, Torres J, Itzkowitz SH. Inflammatory bowel disease and primary sclerosing cholangitis: a review of the phenotype and associated specific features. Gut Liver 2018;12:17-29.

23. Jess T, Rungoe C, Peyrin-Biroulet L. Risk of colorectal cancer in patients with ulcerative colitis: a meta-analysis of population-based cohort studies. Clin Gastroenterol Hepatol 2012; 10:639-645.

24. Zheng HH, Jiang XL. Increased risk of colorectal neoplasia in patients with primary sclerosing cholangitis and inflammatory bowel disease: a meta-analysis of 16 observational studies. Eur J Gastroenterol Hepatol 2016;28:383-390.

25. Weismüller TJ, Trivedi PJ, Bergquist A, et al. Patient age, sex, and inflammatory bowel disease phenotype associate with course of primary sclerosing cholangitis. Gastroenterology 2017;152:1975-1984. 
See "Association of young age and male sex with primary sclerosing cholangitis in Taiwanese patients with inflammatory bowel disease" on pages 213-230.
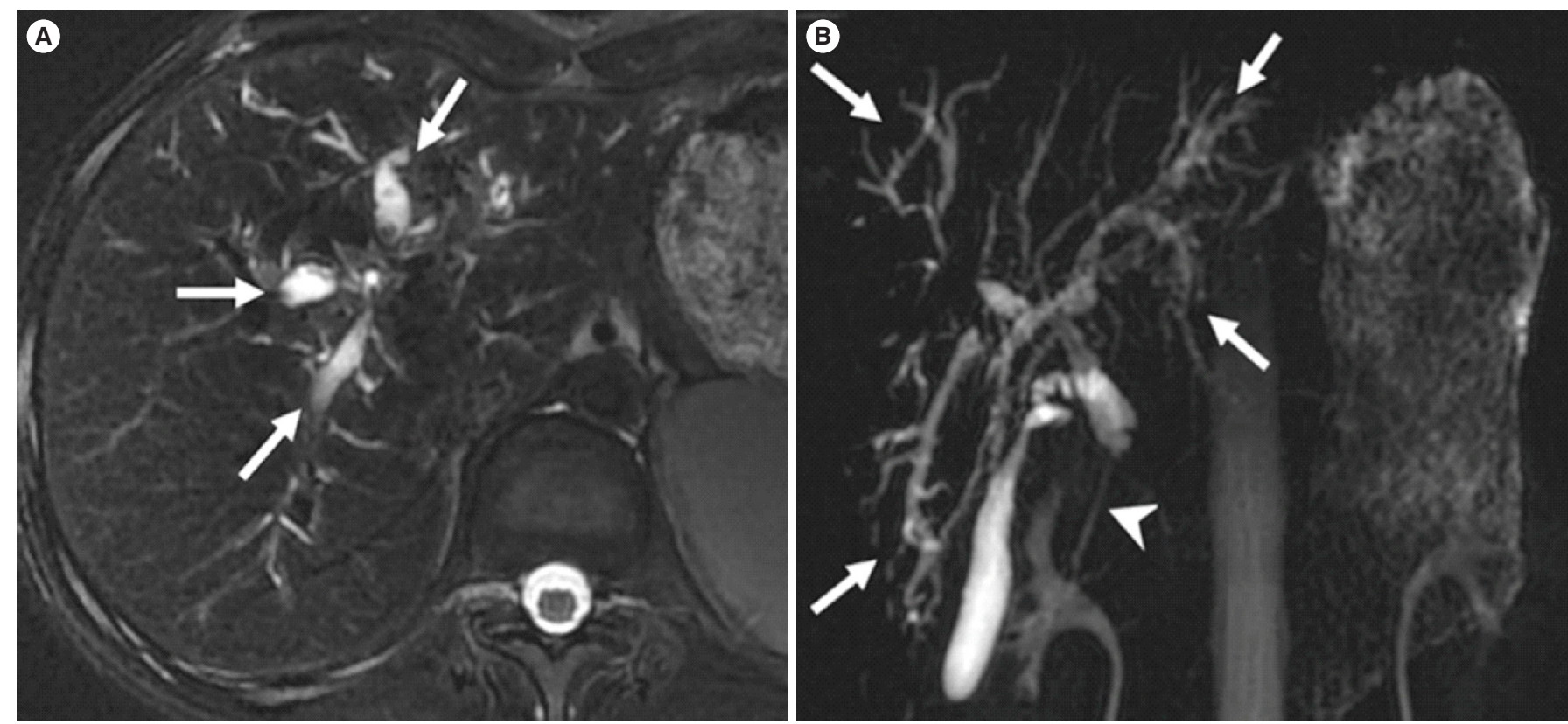

Supplementary Fig. 1. Images of a 24-year-old man with ulcerative colitis and primary sclerosing cholangitis. (A) Axial T2-weighted magnetic resonance imaging with fat suppression shows dilated bilateral intrahepatic bile ducts (arrows) with stenosis at hepatic hilum. (B) Coronal thick slab magnetic resonance cholangiopancreatography shows dilated bilateral intrahepatic bile ducts (arrows) with stenosis at hepatic hilum. There is also a segmental narrowing of the common bile duct (arrowhead). 